\title{
Immunomodulatory effects of probiotic Bifidobacterium bifidum with tacrolimus and sirolimus in mouse skin graft model
}

\author{
Ahram Han ${ }^{1}$, Hyo Kee Kim², Sanghyun Ahn', Seung-Kee Min ${ }^{1}$, Sujeong Kim³ ${ }^{3}$, Chanyeong Jeong ${ }^{3}$, Hansoo Park ${ }^{3}$, Sangil Min ${ }^{1}$, \\ Jongwon $\mathrm{Ha}^{1}$
}

\footnotetext{
${ }^{1}$ Department of Surgery-Transplantation, Seoul National University College of Medicine, Seoul, Korea

${ }^{2}$ Department of Surgery, Korea University Guro Hospital, Seoul, Korea

${ }^{3}$ Department of Biomedical Science and Engineering, Gwangju Institute of Science and Technology, Gwangju, Korea
}

Background: Accumulating evidence suggests that gut microbiota actively crosstalks with the host immune system, and alterations of gut microbiota may exert immune regulatory effects. We aimed to identify probiotic strains capable of modulating the immune response in a mouse allogeneic skin graft model to evaluate its potential as a therapeutic adjunct in the transplantation field.

Methods: Tail skin of BALB/c mice was grafted to the back of C57BL6 mice. Recipient mice were treated with one of five probiotic strains (Lactobacillus lactis, L. fermentum, L. rhamnosus, Bifidobacterium bifidum, and L. reuteri) alone or in conjunction with tacrolimus or sirolimus, and survival of the skin grafts was compared to the control groups with either no medication, tacrolimus without probiotics, or sirolimus without probiotics. The experiment was repeated for selected probiotic strains that showed immunomodulatory effects for quantitative assessment of cytokines using qRT-PCR.

Results: Co-administration of $B$. bifidum with tacrolimus significantly improved skin allograft survival when compared to either the no medication group (mean 17 days vs. 11.7 days, $P<0.001$ by log-rank test) or to the tacrolimus only group (mean 17 days vs. 12.7 days, $P<0.001$ by log-rank test). $B$. bifidum also demonstrated synergistic survival improvement when administered with sirolimus (mean 15.6 days; $P=0.019$ and $P=0.036$ when compared to the control group and the sirolimus only group, respectively). Skin grafts from recipients treated with $B$. bifidum and tacrolimus or $B$. bifidum and sirolimus showed significantly increased expression of anti-inflammatory cytokine, interleukin-10 (IL-10). Expression of proinflammatory cytokine, IL-6 was also markedly inhibited in $B$. bifidum+sirolimus group.

Conclusions: $B$. bifidum promoted allogeneic skin graft survival in mice synergistically with tacrolimus or sirolimus, possibly through induction of IL-10 production. Our study suggests that such a synergistic effect of $B$. bifidum may be applicable as an adjunct to conventional immunosuppressive therapy. 